\title{
BMJ Open How do participatory methods shape policy? Applying a realist approach to the formulation of a new tuberculosis policy in Georgia
}

\author{
Bruno Marchal, ${ }^{1}$ Ibukun-Oluwa Omolade Abejirinde (D) , ${ }^{1}$ Lela Sulaberidze, ${ }^{2}$ \\ Ivdity Chikovani, ${ }^{2}$ Maia Uchaneishvili, ${ }^{2}$ Natia Shengelia, ${ }^{2}$ Karin Diaconu (D) , ${ }^{3}$ \\ Anna Vassall, ${ }^{4}$ Akaki Zoidze, ${ }^{2}$ Ariadna Nebot Giralt, ${ }^{1}$ Sophie Witter (i) ${ }^{3}$
}

To cite: Marchal B, Abejirinde I00 , Sulaberidze L, et al. How do participatory methods shape policy? Applying a realist approach to the formulation of a new tuberculosis policy in Georgia. BMJ Open 2021;11:e047948. doi:10.1136/ bmjopen-2020-047948

- Prepublication history and additional supplemental material for this paper are available online. To view these files, please visit the journal online (http://dx.doi.org/10.1136/ bmjopen-2020-047948).

Received 15 December 2020 Accepted 10 June 2021

A) Check for updates

(c) Author(s) (or their employer(s)) 2021. Re-use permitted under CC BY-NC. No commercial re-use. See rights and permissions. Published by BMJ.

For numbered affiliations see end of article.

\section{Correspondence to} Ibukun-Oluwa Omolade Abejirinde;

i.o.abejirinde@gmail.com

\section{ABSTRACT}

Objectives This paper presents the iterative process of participatory multistakeholder engagement that informed the development of a new national tuberculosis (TB) policy in Georgia, and the lessons learnt.

Methods Guided by realist evaluation methods, a multistakeholder dialogue was organised to elicit stakeholders' assumptions on challenges and possible solutions for better TB control. Two participatory workshops were conducted with key actors, interspersed by reflection meetings within the research team and discussions with policymakers. Using concept mapping and causal mapping techniques, and drawing causal loop diagrams, we visualised how actors understood TB service provision challenges and the potential means by which a results-based financing (RBF) policy could address these. Setting The study was conducted in Tbilisi, Georgia. Participants A total of 64 key actors from the Ministry of Labour, Health and Social Affairs, staff of the Global Fund to Fight AIDS, TB and Malaria Georgia Project, the National Centre for Disease Control and Public Health, the National TB programme, TB service providers and members of the research team were involved in the workshops.

Results Findings showed that beyond provider incentives, additional policy components were necessary. These included broadening the incentive package to include institutional and organisational incentives, retraining service providers, clear redistribution of roles to support an integrated care model, and refinement of monitoring tools. Health system elements, such as effective referral systems and health information systems were highlighted as necessary for service improvement.

Conclusions Developing policies that address complex issues requires methods that facilitate linkages between multiple stakeholders and between theory and practice. Such participatory approaches can be informed by realist evaluation principles and visually facilitated by causal loop diagrams. This approach allowed us leverage stakeholders' knowledge and expertise on TB service delivery and RBF to codesign a new policy.

\section{INTRODUCTION}

Although Georgia has made significant progress in controlling tuberculosis (TB), with
Strengths and limitations of this study

- Participation of a wide range of stakeholders provided a reality check to the initial plans of policymakers and the evaluation design of the researchers.

- Concept mapping and causal mapping strengthened the participatory process of policy formulation.

- Applying realist methodology further informed policymaking by stimulating a reflection on the influence of the policy context.

- A study limitation was the lack of inclusion of participants without a biomedical or health programme background.

- Absence of consistency in attendees from the first to the second workshop limited continuity of the reflection process.

reported success rates of $83 \%$ for new and relapsed $\mathrm{TB}$ cases, some challenges remain, including high rates of drug-resistant TB (DRTB) (11\% incidence in new patients and $31 \%$ in those previously treated) and relatively low cure rates for DR-TB. ${ }^{1}$ Following the collapse of the Soviet Union, several waves of health system reforms were implemented in Georgia from 1994 onwards. These led to a provider-purchaser split whereby the Ministry of Labour, Health and Social Affairs (MoLHSA) took on the role of priority setting, policy formulation, programme development and funds allocation, ${ }^{2}$ while the actual TB programme delivery was relegated to private sector providers. During Soviet times, these functions were administered through the highly centralised Semashko system; healthcare personnel were employed by the state and were redistributed on a hierarchical basis at district, regional and republican levels. The system was tax-based with a guiding principle of granting the population universal access to healthcare free of charge, although 
illegal out-of-pocket payments were extremely common. ${ }^{2}$ In the aftermath of the Rose Revolution (2003-2007), healthcare delivery was further privatised and many disease control components were transferred to a diverse range of private providers, from small rural healthcare facilities to networks of healthcare services. ${ }^{3}$ Although the National TB programme remained responsible for policies, programmes, surveillance and monitoring, TB control and service delivery activities were effectively devolved to private providers.

In 2015, health authorities and TB control programme officials of Georgia, a country with 3.7 million inhabitants in the Caucasus region, initiated the process of developing a new policy to improve the performance of the TB control programme. Policymakers identified the poor integration of TB services at primary care level and low remuneration of service providers as barriers to effective care and treatment of TB patients. ${ }^{4}$ Reports of low coverage and poor adherence to treatment were also linked to low interest of private providers in sustaining TB service provision. Poor motivation of TB professionals further exacerbated the challenges. ${ }^{5}{ }^{6}$ In response, through a concept note submitted to the Global Fund to Fight AIDS, TB and Malaria (GFATM), the Government of Georgia articulated its intention to pilot a resultsbased financing (RBF) scheme to motivate providers and improve TB service delivery in private settings.

Although the concept note indicated the Government's readiness to test this new financing model, it provided little clarity on its design or on the evaluation model to be used. In response, in March 2017, the 4-year RBF for TB care (Results4TB) project was launched as a collaborative project between researchers and national policy makers. This Economic and Social Research Council/Medical Research Council/Department for International Development/Wellcome Trust-funded project brings together policymakers, TB programme managers and national and international researchers to inform the policy formulation, document and evaluate its implementation and assess its cost-effectiveness. The project intends to introduce monetary incentives for health providers, rolled out alongside existing incentive schemes for patients, to improve medication adherence and as such improve treatment success rates for both drug-susceptible and multidrug-resistant TB patients.

The Results4TB project adopted a participatory, codesign approach to developing the study protocol that incorporates an impact evaluation, a cost-effectiveness analysis and a realist research component. We refer to Marchal et $a l^{7}$ for the protocol of the realist component, Chikovani $e t$ al for the trial protocol ${ }^{8}$ and to the project's website ${ }^{9}$ for further information.

Since the publication of guidance on evaluation of complex interventions by the MRC in 2000 and of revised guidance in $2008,{ }^{10}$ trial studies often include implementation fidelity evaluations, process evaluations and/or context mapping exercises. These are often adopted in trials in an effort to enhance understanding of the actual implementation processes and the influence of context on the observed outcomes. ${ }^{11}$ In 2015, the MRC issued guidance on process evaluations of complex interventions, ${ }^{11}$ which was followed by several studies that combined realist process evaluations with trials ${ }^{12-14}$, as well as by calls to go a step further and develop theory-informed trials. ${ }^{15}{ }^{16}$ One innovation the Results4TB project pursues is to test how theory-informed trials can be designed and implemented.

Realist evaluation (RE) is a theory-based approach that is recognised for its usefulness in informing evidenceinformed policy and practice. ${ }^{17}$ Based on a realist philosophy of science, it responds to a need for actionable evidence for decision-making. Rather than merely confirming if policies and programmes work, realist inquiry identifies "what works in which circumstances and for whom?'. ${ }^{18} \mathrm{RE}$ is increasingly used in the field of health policy and systems, ${ }^{19}$ for instance in the study of policy implementation, ${ }^{20}$ leadership and management, ${ }^{21}{ }^{22}$ clinical decision-making support, ${ }^{23}$ adherence to treatment, ${ }^{24}$ and infection control. ${ }^{25}$

Besides including a realist research substudy, the Results4TB project adopted the RE perspective to inform the overall study design. A premise of the project is that this would have a number of advantages. First, the development of a detailed hypothesis in the form of an initial programme theory (IPT). The IPT can best be described as a detailed hypothesis that specifies which mechanisms a programme is expected to trigger among different groups of actors in order to achieve results. It also hypothesises the context conditions under which the programme would work. The IPT thus explains how the programme is expected to generate outcomes and in which conditions. ${ }^{18}$

In the case of Results4TB, we expected RE would stimulate researchers to develop a shared understanding of the policy, its objectives and the way it would work. This in turn would facilitate the integration of project sub-studies on its impact, cost-effectiveness and the underlying processes of implementation and evaluation, contribute to leaner data collection and a more integrated data analysis strategy. ${ }^{26}$ Second, the RE approach can stimulate participation of key actors from the very start: eliciting the assumptions of the actors would both enrich the researchers' understanding of the policy, and also establish strong links with the commissioners, designers, implementers and beneficiaries of the new policy. This engagement would allow existing tacit actor knowledge, evidence and scientific analysis to feed into the policy formulation, leading to a realist formative process. ${ }^{27}$ Through these processes, critical issues and underdeveloped elements can be identified, leading to better intervention planning or implementation, and indirectly to better programme uptake. ${ }^{28}{ }^{29}$ Such participatory processes can also help in better designing the evaluation by making the assumed causal pathways explicit and identifying potential intermediate variables and interim outcomes that may help in monitoring proximal and distal outcomes. ${ }^{30}$ In the field 


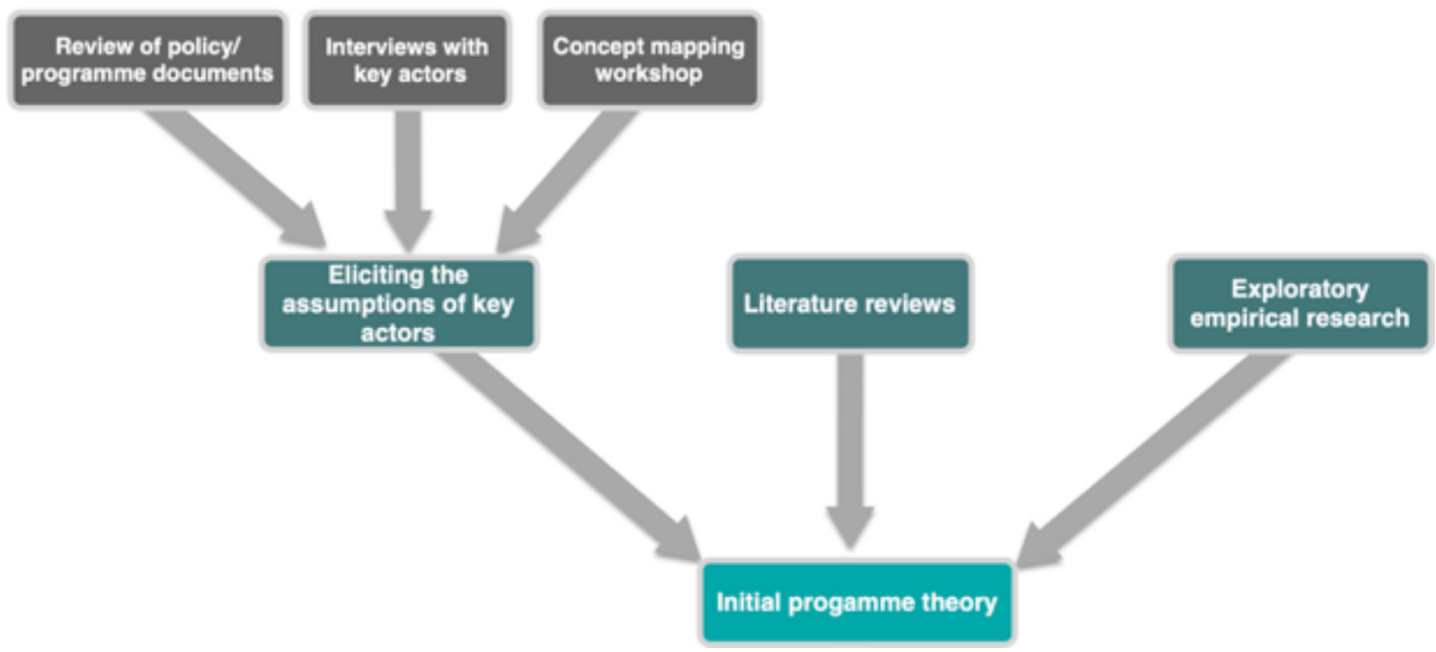

Figure 1 Methods to develop the initial programme theory.

of theory-driven evaluation, group model building techniques or other methods to jointly develop impact pathways or logic models are frequently used. ${ }^{31}{ }^{32}$ To the best of our knowledge, there is little documentation of how such participatory processes are being applied in REs and perhaps even less for simultaneously informing policy formulation and evaluation design within the framework of a trial.

In this paper, we present the iterative process of participatory multistakeholder engagement that we organised at the start of the project to elicit the IPT of the RBF policy. We also discuss the lessons learnt and compare our experience with other projects.

\section{METHODS}

The first step in any realist study is eliciting the IPT. There are generally three ways to elicit the programme theory (figure 1). First, in line with the participatory outlook of RE, the implicit assumptions of the key actors about how the programme is supposed to work can be unearthed. Understanding these assumptions is important since they influence the choices, design and implementation of a programme. ${ }^{18}$ Second, literature reviews can be carried out to identify existing evidence, concepts and theories. ${ }^{29}$ Third, if there is no or little documented evidence, exploratory research can be carried out in lieu of a literature review. ${ }^{33}$

In this project, we elicited the assumptions of key actors regarding the problem of the 'poor' performance of the TB programme through a policy document review, concept mapping workshops and interviews with policymakers (figure 1).

\section{Document review}

We first collected and reviewed National TB Programme regulatory documents, the Strategic Plan for TB control for 2016-2020, TB programme guidelines, WHO mission reports and study reports on TB. The document review focused on the identification of key components of the existing TB control policy in Georgia, its objectives and beneficiaries and on how the TB programme operates throughout the country. ${ }^{34}$ This resulted in a preliminary root cause analysis of the problem.

Table 1 Overview of workshop participants

\begin{tabular}{ll}
\multicolumn{2}{l}{ Number of persons } \\
\hline $\begin{array}{l}\text { First } \\
\text { workshop } \\
\text { (May 2017) }\end{array}$ & $\begin{array}{l}\text { Second } \\
\text { workshop } \\
\text { (August 2017) }\end{array}$ \\
\hline 13 & $6^{\star}$ \\
\hline 5 & 5
\end{tabular}

\begin{tabular}{|c|c|c|}
\hline Stakeholder group & (May 2017) & (August 2017) \\
\hline Research team/CIF staff & 13 & $6^{*}$ \\
\hline $\begin{array}{l}\text { National Centre for Disease } \\
\text { Control and Public Health } \\
\text { (NCDC) }\end{array}$ & 5 & 5 \\
\hline $\begin{array}{l}\text { Ministry of Health, Labour and } \\
\text { Social affairs (MoHLSA) }\end{array}$ & 2 & 2 \\
\hline $\begin{array}{l}\text { Country Coordination } \\
\text { Mechanism (CCM) } \\
\text { Representative }\end{array}$ & 1 & 1 \\
\hline TB doctor & 3 & 4 \\
\hline $\begin{array}{l}\text { Civil Society Organisation } \\
\text { (CSO) representative }\end{array}$ & 1 & 1 \\
\hline Health facility managers & 2 & 4 \\
\hline TB regional coordinator & 1 & 1 \\
\hline $\begin{array}{l}\text { Directly Observed Therapy } \\
\text { (DOT) nurse }\end{array}$ & 2 & 2 \\
\hline Epidemiologist & 1 & 1 \\
\hline $\begin{array}{l}\text { National Centre for } \\
\text { Tuberculosis and Lung } \\
\text { Diseases } \\
\text { (NCTLD), Tbilisi }\end{array}$ & 0 & 2 \\
\hline Family doctor & 0 & 3 \\
\hline \multirow[t]{2}{*}{ Social service agency } & 0 & 1 \\
\hline & 31 & 33 \\
\hline
\end{tabular}

CIF, Curatio International Foundation; TB, tuberculosis. 


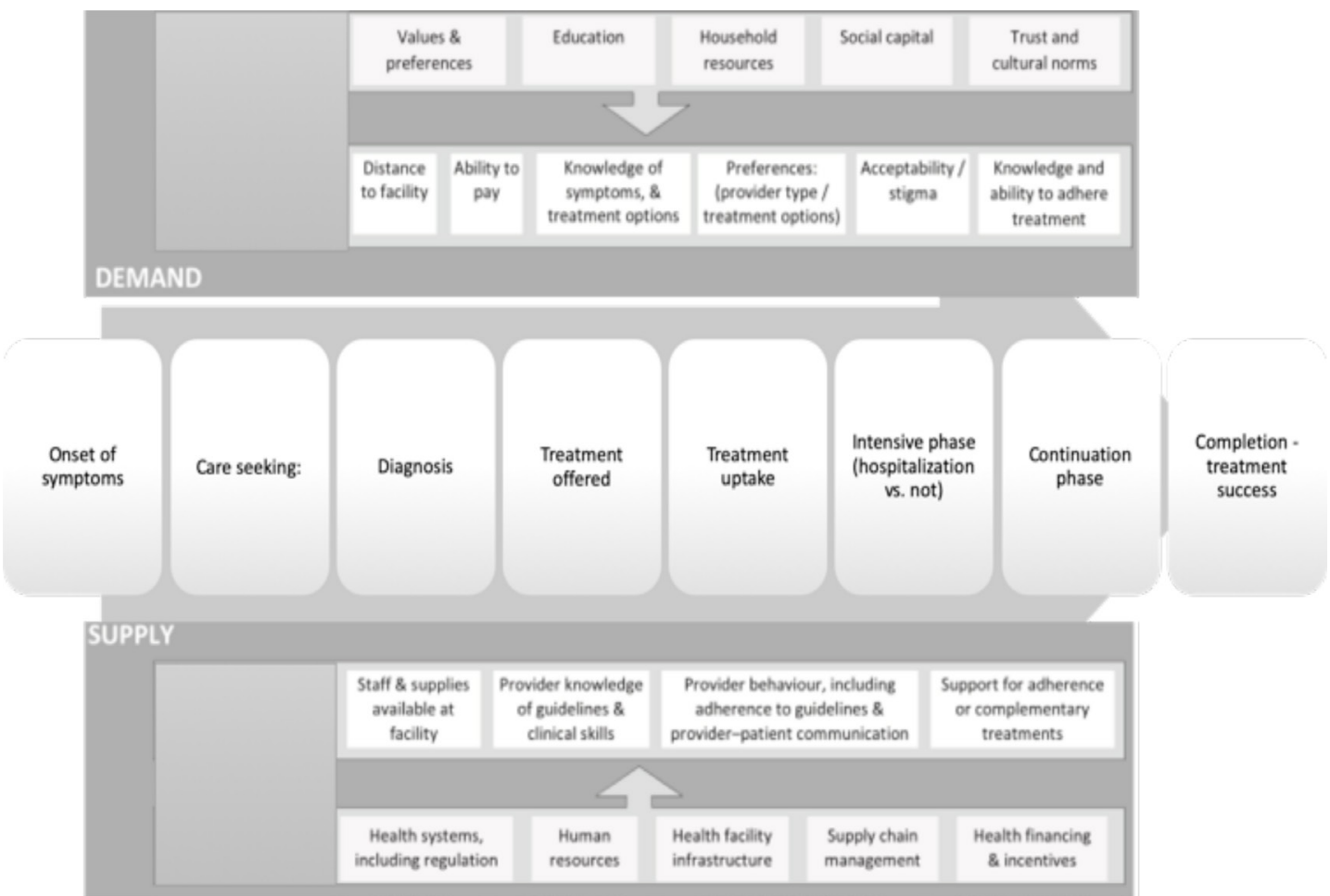

Figure 2 Conceptual framework (adapted from Vassall et al). ${ }^{35}$

\section{First concept mapping workshop}

We conducted two concept mapping workshops with key actors from the MoLHSA, staff of the GFATM Georgia Project, the National Centre for Disease Control and Public Health (NCDC), the National TB programme and with TB service providers. Participants were grouped in a way that ensured stakeholder representation, and that facilitated a 'confrontation' of different actors' perspectives (service managers, providers, programme managers, policymakers, researchers, representatives of funding agency).

In the first workshop, in May 2017, six members of the Georgian research team facilitated the group dialogues. Table 1 presents the background of the 31 participants.

Participants were taken through three steps: (1) identifying the key elements of the problem (identification and analysis of the bottlenecks); (2) identifying the solutions (pathways of change) and (3) identifying the priorities for TB control. Figure 2 presents the conceptual framework, adapted from Vassall $e t a l,{ }^{35}$ that was used to guide the group dialogues. Also see online supplemental file 1.

\section{Interviews with key respondents}

After the first workshop, we carried out unstructured interviews with nine key stakeholders, including policymakers, funders, implementers and programme managers involved in the policy formulation. The main objective was to validate the issues raised during the first workshop and to identify potential solutions. Although no formal interview guide was used, the facilitation probes from the first workshoponline supplemental file 1 guided this informal inquiry (online supplemental file 1). Essentially, we aimed to explore their ideas and assumptions regarding different aspects of the policy. For example, on the need to improve collaboration between TB doctors and family doctors to encourage an integrated care approach, the distribution of roles in the proposed integrated care team, the policy verification system and the data availability for tracking process and outcome indicators.

Questions on TB and integration into primary healthcare (PHC) inquired on side effects and management of comorbidities-which have been raised as important issues affecting overall TB treatment care success rates. Respondents shed light on challenges to service integration, opportunities for improvement and the role of various actors at the different levels of care. Questions on intervention verification aimed to gain insight on existing health information systems collecting TB programmatic data, their weaknesses and opportunities for addressing these, including how to deter fraudulent practices within the system.

Due to the position of the respondents, interviews were not recorded nor systematically transcribed. Rather, notes were taken to inform the revision of the intervention description. Based on the information collected through these discussions, the intervention design was updated, documented, and later on presented to the second workshop participants for further discussion with a wider group of stakeholders.

\section{The second workshop}

The second workshop was organised in August 2017 and was facilitated in the same manner (see online 
supplemental file 2). It was attended by 28 representatives and five research team members, split in two groups. Of those in attendance, five persons in each of the two groups had not attended the first workshop. These included a representative from the Social Service Agency (the State service purchaser under the MoLHSA) and family doctors and managers from primary care facilities (see table 1). During this workshop, participants were asked to analyse the main elements of the proposed new national TB policy - which had been redrafted after the first workshop, focusing on (1) the policy's causal pathway, (2) the underlying processes of change (ie, mechanisms) and (3) the required context conditions.

\section{Analytical strategy}

After the first workshop, we organised a preliminary analysis meeting involving the research team, following which results from the workshop were summarised into tables. As mentioned above, we adopted a realist approach. RE offers the researcher a methodological approach that allows the discernment of how interventions work by exploring mechanisms underlying outcomes and the contextual conditions in which these occur. We used concept mapping and causal mapping techniques ${ }^{36} 37$ to analyse how the actors presented their understanding of the challenges of TB service provision and the potential mechanisms or means by which the policy could address these. Our exploration of mechanisms focused on those underlying the uptake of the policy by providers and managers, assuming that these two groups of stakeholders would have a major influence on actual policy implementation. Both intrinsic and extrinsic motivation drivers were explored-the latter including remuneration, staffing levels and general working conditions. 'Context' was represented by facility-related factors (eg, scale, resource availability, geographical location, quality of care provided) and broader cultural and social factors. Results from the interviews and the document review were used to triangulate results of the workshop. The research team drew on their collective expertise in TB programmes, RBF, clinical care, the Georgian context and health systems. This analysis resulted in causal loop diagrams (CLD) structured to aid visualisation of the patient pathway for TB care within the health system (figure 2). These initial diagrams were reiteratively refined during extensive discussions within the research team and subsequently framed as an IPT (see results section).

After the second workshop, a similar process was carried out, leading to a refined CLD, a reformulated initial programme theory and a new policy package proposal (see results section). Figure 3 depicts the various processes that were combined to develop the IPT of the TB policy and the policy package proposal.

\section{Patient and public involvement}

Public engagement was critical to the redesign of the government policy to improve TB care, because it would give room for the issues experienced by various actors in the health system to be voiced and potentially addressed. While TB patients were not involved in the workshops, there was representation from civil society. The policy formulation workshops allowed for confrontation of perspectives between different actors including service managers, providers, programme managers, policymakers, researchers, representatives of funding agency without whose input there would not have been a revised and robust policy.

\section{Data sharing agreement}

No additional data available.

\section{RESULTS}

In this section, we present the results of the concept mapping workshops and interviews with key respondents,

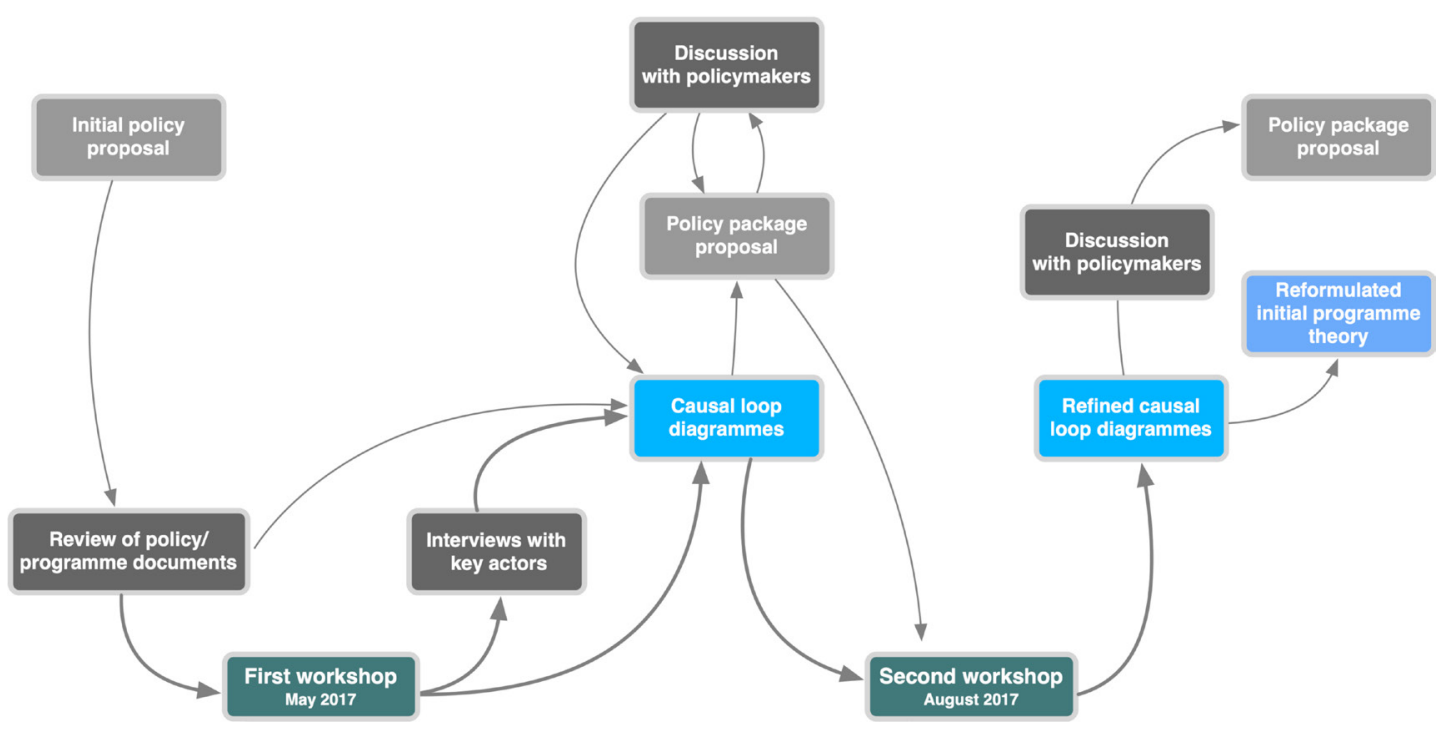

Figure 3 Stages of eliciting the initial programme theory and development of the policy package. 
Table 2 Current bottlenecks and problems, and solutions proposed during the workshops

\section{Current bottlenecks and problems}

Low patient awareness, contributing to which delays in seeking care. High cost of the first visit for patients not referred by a family doctor. Inappropriate management of the side-effects of the TB treatment and associated costs.

Complexity of the directly observed therapy regimen.

Inadequate coordination between providers for the management of comorbidities/side-effects.

\section{Proposed solutions}

Better integration of TB care in general health services. Training of first-line providers and specialists. Institutional and team-based incentives.

Better patient management information systems.

Providing motivation for poorly remunerated and ageing TB providers.

TB, tuberculosis.

and how these contributed to the formulation of the IPT and the policy package.

\section{The first workshop}

During this workshop, participants first identified the current bottlenecks and problems with the National TB programme. These included low patient awareness, which delays patients seeking care, the high cost of the first visit for patients if they are not properly referred by a family doctor, inappropriate management of the sideeffects of the TB treatment and associated high costs, the complexity of the directly observed therapy regimen, as well as inadequate coordination between providers (ie, TB doctors and family physicians) for the management of comorbidities/side-effects and the efficient use of services covered under the Universal Health Coverage programme in this process (table 2)

In the second phase of the workshop, participants moved from bottleneck analysis to the identification of solutions they thought would need to be integrated in the new policy package. The following solutions were identified: better integration of TB care in general health services, training of first-line providers and specialists, institutional and team-based incentives instead of individual incentives, better patient management information systems and motivating poorly remunerated and ageing TB providers (table 2).

After the workshop, we identified the concepts mentioned or referred to by the participants in the discussions. We mapped these on the framework we adapted from Vassall et $a l^{35}$ (figure 2), adding the layer of 'facility managers' and 'providers'. Next, we expanded this concept map into a CLD, which allowed us to identify context factors, potential negative effects and causal relations. Finally, we 'pulled' the diagram to a higher level of abstraction by grouping the causal loop nodes into the categories of policy, actors, context factors, outcomes and mechanisms. The resulting IPT indicated where the new policy would interact with the clinical pathway and how it could contribute to the TB programme outcomes of treatment success, treatment failure and loss to follow-up. Unsurprisingly perhaps, we found that the policy as proposed did not address any demand-side issues (figure 4).

The narrative version of the IPT is presented in box 1 .
The results of the first workshop informed the policy formulation process at the national level. The Curatio International Foundation (CIF) team and international team members were involved in drafting the intervention concept and held several meetings with representatives of the NCDC and the MoLHSA. In essence, the research team argued that there was a need for a comprehensive package, including performance-based incentives for teams as opposed to incentivising only individual providers, a reallocation of roles and responsibilities of healthcare providers (including family doctors) in the provision of TB care, the implementation of new tools (including patient management plans) and training for specialists on the management of side effects. These elements were taken into account by the policymakers. The inputs from the first workshop thus contributed to the formulation of a new TB policy package (figure 5).

\section{Second workshop}

At the start of this workshop, the CIF team presented the updated policy package, its objectives and the implementation modalities. The participants first discussed how they expected the policy would result in better TB control in the long term. To do so, they discussed the policy's causal pathway, starting from components of the policy package and from the beneficiaries to expected effects and potential negative effects. Where opinions diverged, workshop facilitators stimulated further exploration of the issue. Participants proposed some modifications of the incentives package, for instance, calling for a refined bonus system. However, they did not challenge the main thrust of the proposed policy package.

In a second step, participants were asked to identify the mechanisms of change (ie, the processes that explain why a change would occur at the level of providers and patients, the healthcare facility or the health system), the essential conditions for the policy to succeed, the contextual constraints and finally, the conditions necessary for a sustained effect. Box 2 presents the key points raised by the stakeholders.

The second workshop resulted in fine tuning the policy package (figure 6). 


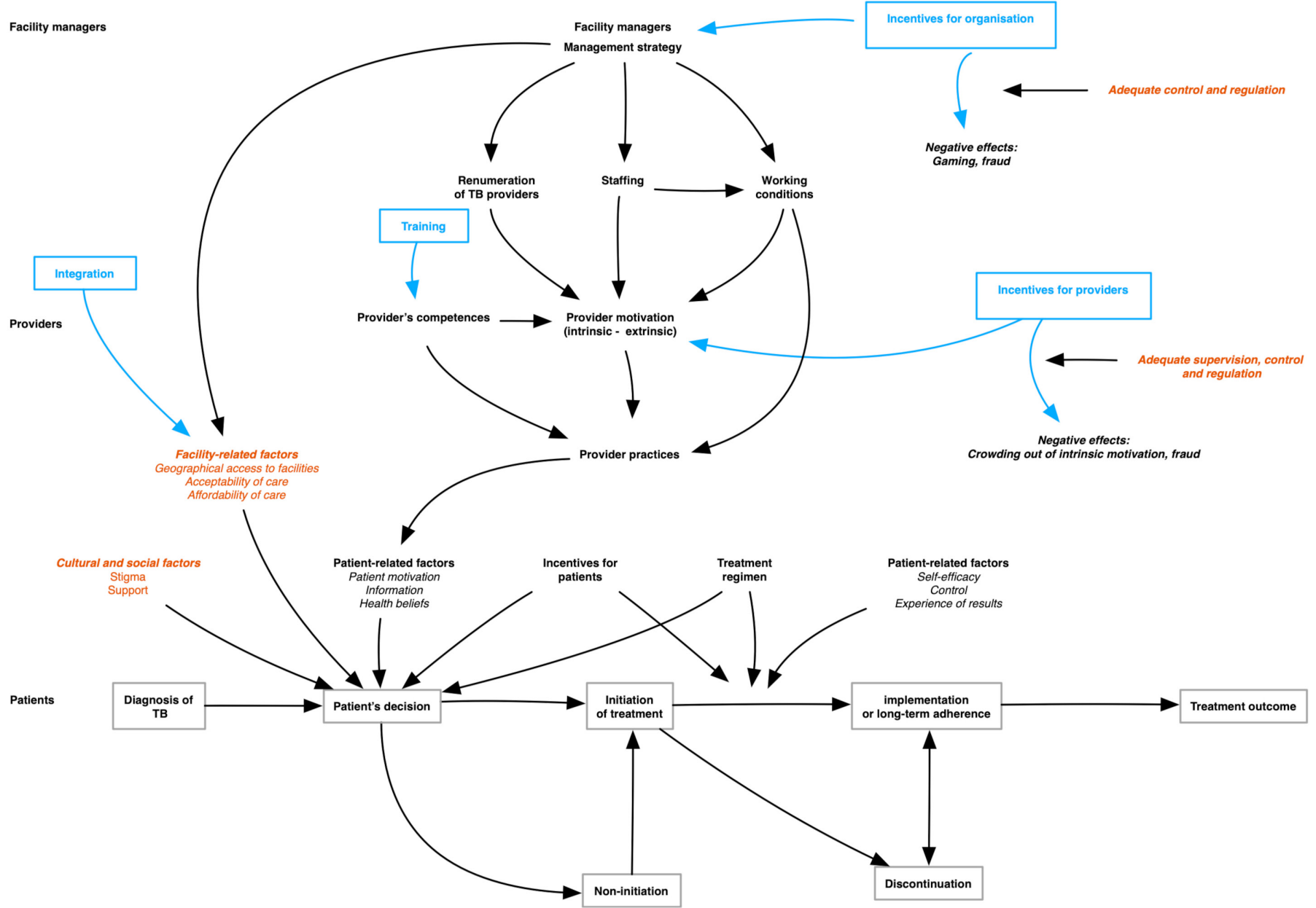

Figure 4 The initial programme theory resulting from the first workshop. TB, tuberculosis.

\section{Final analysis}

In terms of research, the results from the second workshop were incorporated and mapped onto the initial CLD (figure 7). This exercise helped us in formulating a final version of the IPT (box 3).

Analysis from the second workshop also led to revision of some elements of the policy package following discussions with the policymakers. These included defining functions

\section{Box 1 Narrative version of the initial programme theory} (first workshop)

Incentives targeting the organisational level would incite service managers to increase the motivation of their providers, while training would improve the latter's competences.

- Incentives for providers would increase their extrinsic motivation but could lead to crowding out their intrinsic motivation.

- All in all, a change of provider practices would occur, which in turn would affect how patients would take decisions regarding care for their health problem. Their decisions would also be influenced by context factors such as accessibility of the facility, and the acceptability and affordability of tuberculosis care.

- Potential negative effects include gaming, fraud and crowding out of intrinsic motivation, which would be influenced by the degree of supervision, control and regulation. of each type of provider involved in the scheme, reporting requirements, distribution of bonus payment scheme between team members, monitoring and verification. The process was followed by corresponding changes to the design of the Results4TB pilot phase, the implementation manual and other supporting documents (eg, guidelines for Results4TB programme managers, reporting forms to the Social Service Agency, the patient management plan).

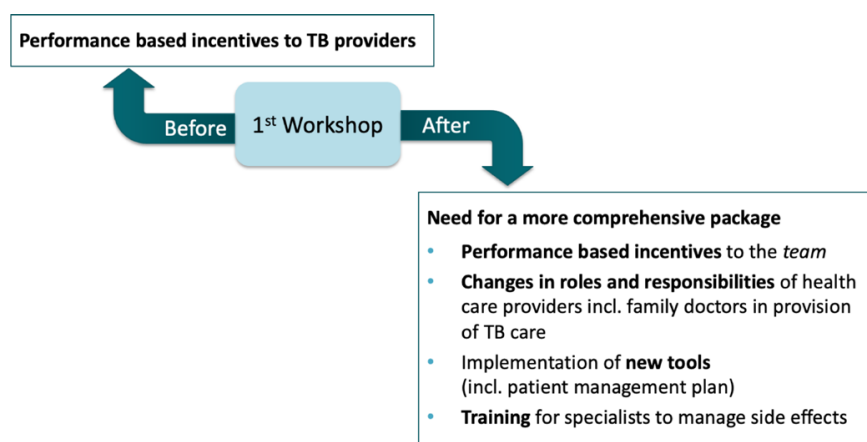

Figure 5 Changes in the policy package after the first workshop. TB, tuberculosis. 
Box 2 Summary of key points raised during the second workshop

- Every tuberculosis (TB) patient should be assigned to a primacy healthcare (PHC) facility with an integrated TB unit where the patient will receive general care from a family doctor as well as TB care by a TB specialist. While this could create geographical barriers for some patients, it would ensure an integrated approach.

- An integrated patient-centred TB care team should link patients to social services as needed.

- The role and functions of rural doctors should be well defined and different bonus payment schemes should be created for them.

- Incentives for family doctors should be a fixed top up to their salary as opposed to incentives per patient.

- Facilities with low numbers of patients need stronger incentives than facilities with a higher patient load.

- Managers of health facilities should ensure adequate service provision (eg, laboratory investigations, consultant visits) through contracts with other PHC facilities if they are unable to offer these services on site.

- The RBF policy and intervention should be incorporated as a component of the National TB Programme.

- The TB state programme budget should be revised to ensure adequate funding for routine tests and investigations. Mechanisms for distributing drugs to manage the side-effects of TB treatment should also be provided for within the TB programme's budget.

\section{DISCUSSION}

In this paper, we set out to report the process of eliciting the IPT of a new RBF policy that the Georgian authorities hope will address the persisting burden of $\mathrm{TB}$ in Georgia and improve service delivery. The Results4TB project took the opportunity of being asked to participate in the formulation and evaluation of the new policy to develop and implement a theory-informed trial design. Our assumption was that applying RE to the process from the very start would both contribute to develop a shared understanding of the policy among the researchers (and thus a theory-informed study design), and also allow us to identify the assumptions of the key actors, including the research team, and thus inform the policy package leveraging the strengths of diverse stakeholder perspectives.

The workshops proved helpful in informing the policy content and implementation design. The active

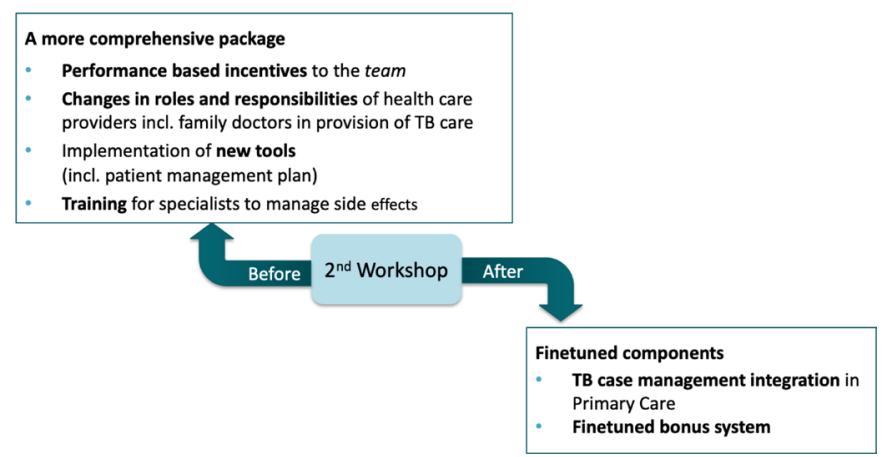

Figure 6 Changes in the policy package after the second workshop. TB, tuberculosis. involvement of key stakeholders allowed tacit knowledge and expertise on TB service delivery and RBF from a range of stakeholders to be leveraged in codesigning the policy. Similar benefits of a participatory approach have been reported in health and non-health policymaking processes. ${ }^{38} 39$ Similarly, the workshops allowed for a bottom-up problem analysis whereby participants working at operational level provided feedback based on their daily experience. ${ }^{38}$ Workshop participants indicated that beyond provider incentives, additional policy components would be necessary to achieve desired goals. This included training of service providers to increase family doctors' awareness on TB case detection, organising proper referral to $\mathrm{TB}$ services, better management of sideeffects, a redistribution of roles between the PHC and TB specialised services and between care team members to support an integrated care model, training of integrated care team members on the principles of the new policy, creating a 'patient card' to increase patient involvement in TB care management and refinement of policy monitoring tools. These were issues not previously considered and could have led to programme failure if unaddressed.

To facilitate discussions during the first workshop, we adopted the patient pathway of Vassall et $a l^{35}$ which proved useful in structuring the analysis. Participants were able to identify crucial issues which did not appear in the framework, including service organisation gaps, MoLHSA programme issues and social support-all factors impeding the delivery of $\mathrm{TB}$ care which the proposed policy would need to take into account.

CLDs were drawn with the aim of integrating findings from the workshops and the ideas of the researchers between the first and second workshops. We used CLDs to develop a graphical representation of the patient and health system pathways and the barriers and facilitators to the policy, more than to identify feedback loops as was done for instance by Renmans $e t a l^{40}$ and Kwamie $e t a l^{22}$ The CLDs demanded systematic in-depth discussions among the researchers and stimulated them to make their assumptions explicit, which has been mentioned as a key strength of CLD development. ${ }^{41}{ }^{42}$ We also used the CLDs to identify key health system elements for successful TB care and used them to identify specific factors that would drive TB service integration at various levels. A similar use of CLDs was reported by Renmans et al. ${ }^{40}$ While we did not (yet) exploit the CLDs to their full potential, the CLD development ultimately contributed to developing a more refined IPT and gave a visual overview that all stakeholders could appreciate and contribute to.

The research process we adopted is an example of embedded research. A senior researcher of the CIF team for instance played the dual role of being senior researcher, and a member of Parliament of Georgia and head of its Health and Social Issues Committee. Embedded research arguably facilitates the uptake of evidence in health policymaking. ${ }^{43}$ In our case, close interaction between policymakers and researchers helped both to refine the research design and also the actual policy. However, other factors 


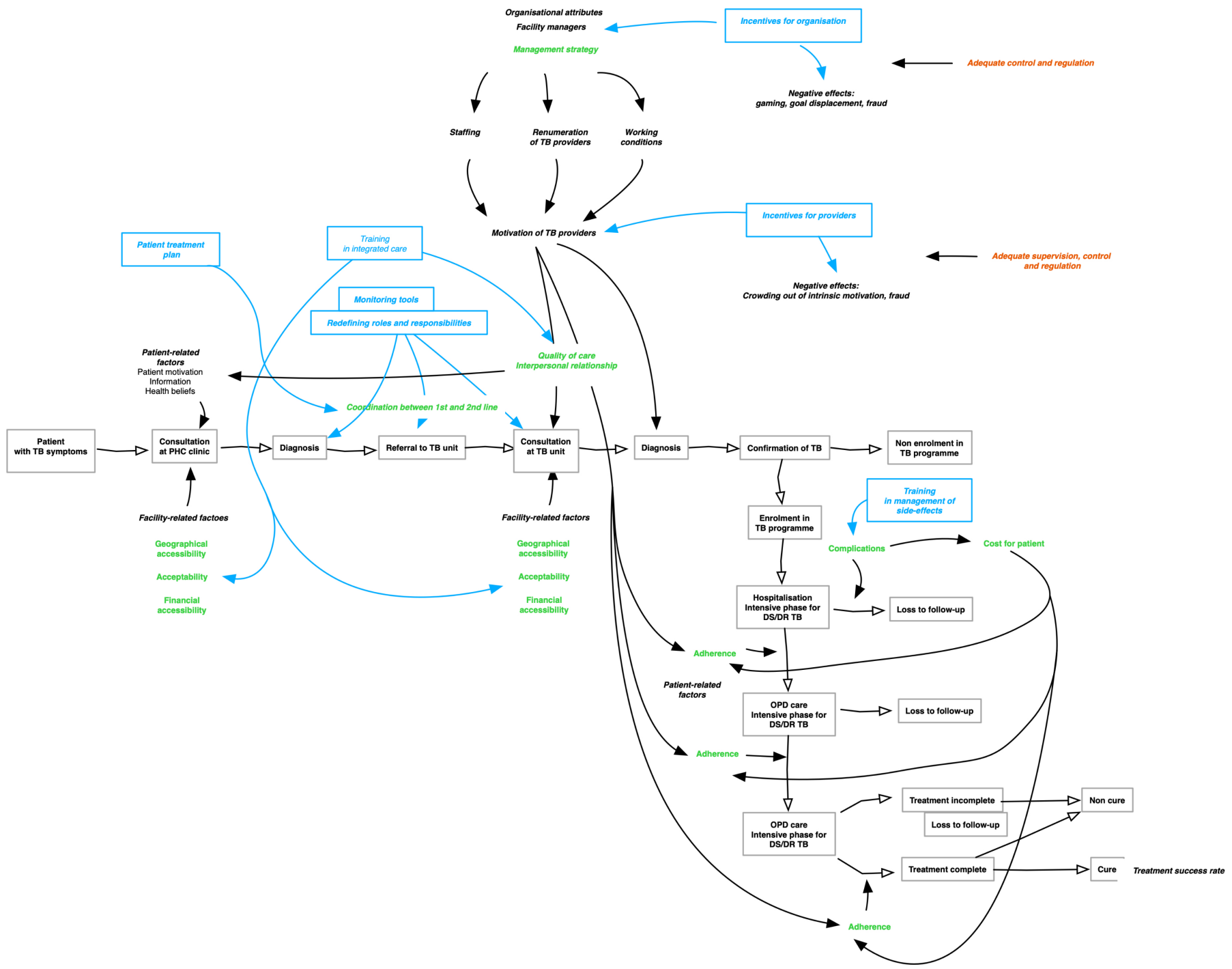

Figure 7 The initial programme theory resulting from the second workshop. DR, drug-resistant; DS, drug-susceptible; TB, tuberculosis.

shaped the actual formulation of the policy. The workshops, for example, played a critical role in identifying existing challenges in TB care management throughout the patient pathway from a bottom-up perspective. The first workshop led to the 'discovery' of contextual factors influencing TB treatment outcomes that were unique and/or specific to the Georgian context and which we did not identify in the literature reviews. One such factor is the lack of information sharing between general and specialised TB service providers. The second workshop contributed to examining the operational constraints to a successful policy implementation (eg, difficulties to develop a monitoring system caused by the nonfunctional TB electronic reporting module) and propose appropriate solutions. Embedded research has a number of conditions of success including the need to have a flexible approach and the ability to navigate contextual realities, including constraints. ${ }^{44}$

By taking a participatory approach, both workshops created an atmosphere where the inputs of all participants were valued and acknowledged as critical for designing the new TB policy. This approach eventually resulted in the creation of a more context-adapted policy package. All critical feedback received during the workshops was later used by the research team to refine the policy concept which led to the various policy documents, including the programme component of the RBF pilot phase, the implementation manual and other supporting documents to facilitate a smooth policy introduction. The limited time allocated to group work during the workshops did not facilitate in-depth discussions on some topics, like the quality of the evidence provided by participants to make a point. ${ }^{27}$

This study has some limitations. Ideally, both workshops would have been attended by the same participants to ensure continuity of the reflection. However, each workshop had slightly different objectives, and the results of the first workshop were presented at the start of the second one allowing for an additive process. We also took care to ensure that participants who were unavailable for the second workshop were replaced by representatives from the same category of stakeholders. 
Box 3 Narrative version of the reformulated initial programme theory

$\begin{array}{lll}\text { Financial incentives } & \text { will stimulate facili- } \\ \text { ty managers to (1) continue providing TB }\end{array}$ services, (2) remit incentives to TB providers within their facilities and (3) optimise their TB service activities (mechanism of organisational self-interest), on the condition that incentives are considered to be adequate and that sufficient monitoring and regulation systems are in place, in the absence of which gaming and fraud could occur.

- Incentives for providers will increase their extrinsic motivation (mechanism) and if the staffing and working conditions are adequate (contextual prerequisite) they will contribute to better care for TB patients (outcome). Motivated and competent providers will provide better adherence support to patients, if supervision, monitoring and regulation are adequate. If not, crowding out of intrinsic motivation and gaming may occur (unintended consequence).

- Training for providers (eg, on side effect of drugs, management of comorbidities and patient-centred care as well as on how the RBF scheme will work) will enhance their competence, which leads to higher self-efficacy (mechanism). The latter, combined with an increase in extrinsic motivation, contributes to behaviour change, which will enable patients to better adhere to the treatment (outcome).

- TB patients who are informed, motivated and supported by providers, and trained to develop the needed skills, will initiate adherence if the social and cultural context is favourable (eg, absence of stigma), if the facility is accessible, acceptable and affordable and if they consider the treatment regime as acceptable.

- The integration of TB care will improve the continuum of care between general and specialised TB services and thus facilitate the patient to correctly follow the treatment and care pathway towards being cured.

- TB patients who experience positive effects, feel capable of managing their health condition (mechanism of self-efficacy) and feel in control of decisions about their care are more likely to maintain long-term adherence, on the condition that they consider the treatment regime to be acceptable.

A second issue concerns the selection of stakeholders: while it was quite wide ranging in terms of including representatives from all levels of the health system, most attendees had a biomedical or health programme background with limited representation from the community. We attempted to counter this professional bias by encouraging reflexion and critique, and used techniques to reduce group think as much as possible, for instance, always starting analysis with individual tasks, or by having facilitators challenging statements. In the next phases of the Results4TB programme, patient groups and community representatives will be engaged prior to actual policy trial.

Further research into participatory policymaking could explore the conditions in which more inclusive participation occurs and the role herein of providing safe spaces for participants from different hierarchical levels to speak out, or of ensuring that all types of information and evidence are considered.

\section{CONCLUSION}

Developing policies that address complex issues, such as TB care in Georgia, and conducting policy research requires methods that facilitate the linkages between multiple stakeholders including researchers, and between theory and evidence on the one hand and practice on the other hand. Adopting a participatory approach to developing the IPT and the policy package, we found that the realist methodology helped us to leverage the tacit knowledge and expertise on TB service delivery and RBF of a wide range of stakeholders to codesign the policy. The policy now reflects a more complex intervention, which will be tested using a combination of realist, trial and costeffectiveness tools.

\section{Author affiliations}

${ }^{1}$ Health Systems and Health Policy Research Group, Department of Public Health, Institute of Tropical Medicine, Antwerpen, Belgium

${ }^{2}$ Research Unit, Curatio International Foundation, Tbilisi, Georgia

${ }^{3}$ Institute for Global Health and Development, Queen Margaret University Edinburgh, Musselburgh, UK

${ }^{4}$ Department of Global Health and Development, London School of Hygiene \& Tropical Medicine, London, UK

Twitter Ibukun-Oluwa Omolade Abejirinde @lade_abeji and Sophie Witter @ sophie_witter

Acknowledgements We would like to acknowledge the inputs of all workshop participants, which were essential for developing the programme theory as well as the policy package.

Contributors BM, LS and IC conceptualised the manuscript with input from I-OOA, MU, NS, KD, AV, AZ, ANG and SW, who collectively designed and executed the research study as members of the Results4TB research consortium. The participatory workshops were mainly facilitated by the research consortium and the CIF team (LS, IC, MU, NS and AZ) in particular. I-OOA and BM prepared the drafts of the article with additional contribution from the other authors (LS, IC, MU, NS, KD, $A V, A Z, A N G$ and SW). All authors contributed to and agreed on the final manuscript.

Funding The Results4TB study is a 4-year (2017-2021) project funded under the Health Systems Research Initiative call 3 of the MRC/ESRC/DfID/Wellcome Trust consortium (Grant Ref: MR/P015018/1).

Competing interests $\mathrm{AZ}$ being the project PI, is both a member of the Results4TB research team and a member of parliament in Georgia.

\section{Patient consent for publication Not required.}

Ethics approval The Results4TB trial protocol has been approved by the respective Institutional Review Boards (IRB) of the partner institutions. The realist evaluation component which forms the basis of this manuscript was granted ethical clearance by IRBs of the National Centre for Disease Control and Public Health in Georgia (ref. IRB \# 2018-019) and the Institute of Tropical Medicine, Antwerp (ref. IRB \#- 1240/18).

Provenance and peer review Not commissioned; externally peer reviewed.

Data availability statement All data relevant to the study are included in the article or uploaded as supplemental information.

Supplemental material This content has been supplied by the author(s). It has not been vetted by BMJ Publishing Group Limited (BMJ) and may not have been peer-reviewed. Any opinions or recommendations discussed are solely those of the author(s) and are not endorsed by BMJ. BMJ disclaims all liability and responsibility arising from any reliance placed on the content. Where the content includes any translated material, BMJ does not warrant the accuracy and reliability of the translations (including but not limited to local regulations, clinical guidelines, terminology, drug names and drug dosages), and is not responsible for any error and/or omissions arising from translation and adaptation or otherwise.

Open access This is an open access article distributed in accordance with the Creative Commons Attribution Non Commercial (CC BY-NC 4.0) license, which permits others to distribute, remix, adapt, build upon this work non-commercially, 
and license their derivative works on different terms, provided the original work is properly cited, appropriate credit is given, any changes made indicated, and the use is non-commercial. See: http://creativecommons.org/licenses/by-nc/4.0/.

\section{ORCID iDs}

Ibukun-Oluwa Omolade Abejirinde http://orcid.org/0000-0003-0139-0541

Karin Diaconu http://orcid.org/0000-0002-5810-9725

Sophie Witter http://orcid.org/0000-0002-7656-6188

\section{REFERENCES}

1 World Health Organization. WHO global tuberculosis database. Available: https://www.who.int/tb/country/data/download/en/ [Accessed 24 Oct 2020].

2 Amiran G, Atun R, Gotsadze G. Health care systems in transition: Georgia. Copenhagen, Denmark World Health Organization, Regional Office for Europe, European Observatory on Health Systems and Policies; 2002.

3 Zoidze A, Rukhadze N, Chkhatarashvili K. Health insurance for poor: Georgia's path to universal coverage.

4 Country Coordination Mechanism (CCM). National strategic plan for tuberculosis control in Georgia 2016-2020. Tbilisi: Ministry of Labor, Health and Social Affairs of Georgia, United States' Agency for International Development (USAID), 2015.

5 Curatio International Foundation. Barriers and facilitators to adherence to treatment among drug resistant TB patients in Georgia, 2016. Available: http://curatiofoundation.org/factors-that-enhanceor-hinder-treatment-adherence-among-drug-resistant-tb-patients-ingeorgia/ [Accessed 24 Oct 2020].

6 World Health Organization. Extensive review of tuberculosis prevention, control and care in Georgia, 6-14. Denmark: WHO Regional Office for Europe, 2014. https://www.euro.who.int/_data/ assets/pdf_file/0008/321947/Extensive-review-TBC-preventioncontrol-care-Georgia-November-2014.pdf

7 Marchal B, Giralt AN, Sulaberidze L, et al. Designing and evaluating provider results-based financing for tuberculosis care in Georgia: a realist evaluation protocol. BMJ Open 2019;9:e030257.

8 Chikovani I, Diaconu K, Duric P, et al. Addressing challenges in tuberculosis adherence via performance-based payments for integrated case management: protocol for a cluster randomized controlled trial in Georgia. Trials 2019;20:536.

9 Curatio International Foundation. Results for Tuberculosis. Results4TB. Available: http://results4tb.curatiofoundation.org/ [Accessed 24 Oct 2020].

10 Anderson R. New MRC guidance on evaluating complex interventions. BMJ 2008;337:a1937.

11 Moore GF, Audrey S, Barker M, et al. Process evaluation of complex interventions: medical Research Council guidance. BMJ 2015;350:h1258.

12 Rycroft-Malone J, Seers K, Eldh AC, et al. A realist process evaluation within the facilitating implementation of research evidence (FIRE) cluster randomised controlled international trial: an exemplar. Implement Sci 2018;13:138.

13 O'Brien R, Buston K, Wight D, et al. A realist process evaluation of enhanced triple $\mathrm{P}$ for baby and mellow bumps, within a trial of healthy relationship initiatives for the very early years (THRIVE): study protocol for a randomized controlled trial. Trials 2019;20:351.

14 Jeon Y-H, Simpson JM, Low L-F, et al. A pragmatic randomised controlled trial (RCT) and realist evaluation of the interdisciplinary home-bAsed Reablement program (I-HARP) for improving functional independence of community dwelling older people with dementia: an effectiveness-implementation hybrid design. BMC Geriatr 2019;19:199.

15 Rycroft-Malone J, Seers K, Chandler J, et al. The role of evidence, context, and facilitation in an implementation trial: implications for the development of the PARIHS framework. Implement Sci 2013;8:28.

16 Stern E, Stame N, Mayne J. Broadening the range of designs and methods for impact evaluations. Institute for Development Studies, 2012.

17 Pawson R. Evidence-Based policy: a realist perspective. SAGE Publications Ltd, 2006.

18 Pawson R, Tilley N. Realistic evaluation. SAGE Publications Ltd, 1997.

19 Marchal B, Kegels G, Van Belle S. Doing realist research. London: SAGE Publications Ltd, 2021.

20 Dossou J-P, De Brouwere V, Van Belle S, et al. Opening the 'implementation black-box' of the user fee exemption policy for caesarean section in Benin: a realist evaluation. Health Policy Plan 2020;35:153-66.

21 Belrhiti Z, Van Damme W, Belalia A, et al. Unravelling the role of leadership in motivation of health workers in a Moroccan public Hospital: a realist evaluation. BMJ Open 2020;10:e031160.

22 Kwamie A, van Dijk H, Agyepong IA. Advancing the application of systems thinking in health: realist evaluation of the leadership development programme for district manager decision-making in Ghana. Health Res Policy Syst 2014;12:29.

23 Abejirinde I-OO, Zweekhorst M, Bardají A, et al. Unveiling the black box of diagnostic and clinical decision support systems for antenatal care: realist evaluation. JMIR Mhealth Uhealth 2018;6:e11468.

24 Mukumbang FC, Marchal B, Van Belle S, et al. A realist approach to eliciting the initial programme theory of the antiretroviral treatment adherence club intervention in the Western Cape Province, South Africa. BMC Med Res Methodol 2018;18:47

25 Williams L, Burton C, Rycroft-Malone J. What works: a realist evaluation case study of intermediaries in infection control practice. $J$ Adv Nurs 2013;69:915-26.

26 Marchal B, Van Belle S, De Brouwere V, et al. Studying complex interventions: reflections from the FEMHealth project on evaluating fee exemption policies in West Africa and Morocco. BMC Health Serv Res 2013;13:469.

27 Brand SL, Quinn C, Pearson M, et al. Building programme theory to develop more adaptable and scalable complex interventions: realist formative process evaluation prior to full trial. Evaluation 2019;25:149-70.

28 Huey CT. Theory-Driven evaluations. SAGE Publications, Inc, 1994.

29 Lipsey MW, Cordray DS. Evaluation methods for social intervention. Annu Rev Psychol 2000;51:345-75.

30 Birckmayer JD, Weiss $\mathrm{CH}$. Theory-Based evaluation in practice. what do we learn? Eval Rev 2000;24:407-31.

31 Mason P, Barnes M. Constructing theories of change: methods and sources. Evaluation 2007;13:151-70.

32 Breuer E, De Silva MJ, Fekadu A, et al. Using workshops to develop theories of change in five low and middle income countries: lessons from the programme for improving mental health care (PRIME). Int $J$ Ment Health Syst 2014;8:15.

33 Marchal B, van Belle S, van Olmen J, et al. Is realist evaluation keeping its promise? A review of published empirical studies in the field of health systems research. Evaluation 2012;18:192-212.

34 Curatio International Foundation. Tuberculosis system overview in Georgia, 2017. Available: http://results4tb.curatiofoundation.org/wpcontent/uploads/2018/07/TB-System-Overview_2017.pdf [Accessed 24 Oct 2020].

35 Vassall A, Mangham-Jefferies L, Gomez GB, et al. Incorporating demand and supply constraints into economic evaluations in lowincome and middle-income countries. Health Econ 2016;25(Suppl 1):95-115.

36 Rosas SR. Concept mapping as a technique for program theory development: an illustration using family support programs. Am J Eval 2005;26:389-401.

37 Trochim W, Kane M. Concept mapping: an introduction to structured conceptualization in health care. Int J Qual Health Care 2005;17:187-91.

38 van den Hove S. Participatory approaches to environmental policymaking: the European Commission climate policy process as a case study. Ecol Econ 2000;33:457-72.

39 Freebairn L, Atkinson J-A, Kelly PM, et al. Decision makers' experience of participatory dynamic simulation modelling: methods for public health policy. BMC Med Inform Decis Mak 2018;18:131.

40 Renmans D, Holvoet N, Criel B. Combining Theory-Driven Evaluation and Causal Loop Diagramming for Opening the 'Black Box' of an Intervention in the Health Sector: A Case of Performance-Based Financing in Western Uganda. Int J Environ Res Public Health 2017:14:1007.

41 Baugh Littlejohns L, Baum F, Lawless A, et al. The value of a causal loop diagram in exploring the complex interplay of factors that influence health promotion in a multisectoral health system in Australia. Health Res Policy Syst 2018;16:126.

42 Lannon C. Causal loop construction: the basics, 2016. The systems thinker. Available: https://thesystemsthinker.com/causal-loopconstruction-the-basics/ [Accessed 24 Oct 2020].

43 Ghaffar A, Langlois EV, Rasanathan K, et al. Strengthening health systems through embedded research. Bull World Health Organ 2017;95:87.

44 Walley J, Khan MA, Witter S, et al. Embedded health service development and research: why and how to do it (a ten-stage guide). Health Res Policy Syst 2018;16:67. 